\title{
Combined Transmitter Diversity and Multi-Level Modulation Techniques
}

\author{
Slimane Ben Slimane \\ Radio Communication Systems \\ Department of Signals, Sensors, and Systems \\ The Royal Institute of Technology (KTH) \\ Electrum 418, 16440 KISTA, Sweden \\ slimane@ radio.kth.se
}

\begin{abstract}
Orthogonal transmitter diversity such as frequency diversity and time diversity is quite simple to implement and, with optimum signal combining, can take full advantage of fading multipath channels. However, such a scheme has a bandwidth efficiency that decreases inversely with the number of diversity branches making it less attractive in wireless communications applications. This paper considers combined orthogonal transmitter diversity and multi-level linear modulation techniques. The idea is to view the signal constellations of the modulation scheme in an augmented signal space formed by the modulation signal dimension and the number of branches of the transmitter diversity scheme. This augmented signal space provides a good spread for the modulation signal points and can be quite efficient for high-level linear modulation techniques. The obtained results show that this combined scheme, not only improves the system performance in both additive white Gaussian noise and fading multipath channels, but also improves the bandwidth efficiency of orthogonal transmitter diversity.
\end{abstract}

Key words: Orthogonal transmitter diversity, multi-level linear modulation, diversity gain, bandwidth efficiency, wireless communications.

\section{Introduction}

Diversity a very good technique for improving the performance of wireless communication links. Orthogonal Transmitter Diversity (TD) such as frequency diversity and time diversity have some properties that are quite attractive in wireless communications as they can provide a diversity gain without the need for multiple transmit/receive antennas. In general, diversity tries to solve the signal fading problem in wireless communication by transmission of several redundant replicas of the information signal that undergo different multipath profiles. The idea is that the repetition of the information, together with an appropriate combining of the received signals, will greatly reduce the negative effects of the radio channel fading. When the replicas of the same information signal are transmitted over independent fading channels, then the probability that all the signal replicas will fade simultaneously is reduced considerably. With frequency diversity, replicas of the same information signal are transmitted on different frequency carriers giving the possibility to take advantage of the frequency variation of the radio channel.
This can, for instance, be used quite easily in wideband OFDM modulation techniques. With time diversity, replicas of the signal are provided in the form of redundancy in the time domain by means of an error control code together with a proper interleaving scheme. The simplest coding scheme is repetitive coding where the same symbol is transmitted over several different frequencies or different time slots. The advantage of repetitive coding is the detection procedure where after signal combining symbol by symbol detection is still possible (Ali et al., 1989). The drawback is ofcourse the bandwidth expansion which makes the system efficiency decreases inversely with the number of repetitions.

Bandwidth efficiency, power efficiency, and signal reliability are important parameters in wireless communications. Increasing the signal quality or reducing the bit error rate of digital modulated signals in fading multipath channels without any efficiency loss is quite difficult. One possible solution to improve the bandwidth efficiency of orthogonal transmitter diversity techniques is by using multi-level bandwidth efficient modulation techniques such as Quadrature Am- 
plitude Modulation (QAM) and Phase Shift Keying (PSK). Using these linear modulation techniques and by increasing the modulation level one can compensate for some of the bandwidth expansion caused by the transmitter diversity scheme. However, it is well known that linear modulation techniques are not power efficient. Hence, increasing the modulation level will require increasing the transmitted power to ensure the required quality of service of the system. Power efficiency is a basic requirement in wireless communication systems to allow small size devises and long battery life. In such a situation one has to make a compromise between bandwidth expansion and power usage. A second possible solution is to use coded modulation. However, the diversity order of coded modulation is limited by the shortest error event path along its trellis diagram which is, in general, quite small for practical TCM schemes (Biglieri et al., 1991).

In this paper we consider combined transmitter diversity and multi-level bandwidth efficient modulation techniques. The main idea is to try to take advantage of the extra dimension provided by the diversity scheme in improving the power efficiency of the used modulation without altering the diversity order of the system. Considering the overall scheme as one entity we can obtain a transmission scheme than can perform very well in both additive white Gaussian and fading multipath channels. This combining procedure does not increase the complexity at the receiver and consecutive received symbols can still be treated independently. The full performance potential of this scheme is obtained by employing a detector based on the Maximum Likelihood Sequence Estimation (MLSE) algorithm on a symbol-by-symbol basis.

Combined transmitter diversity and multi-level modulation can be used in high data rate wireless communications with Orthogonal Frequency Division Multiplexing (OFDM) to improve the link quality or data coverage. For example, using higher-order constellations than the currently used modulations in the IEEE802.11a and IEEE802.11g compliant systems, we can improve the power efficiency of the system without affecting the efficiency of the system and the operation of the different coding schemes (ETSI, 2000), (ETSI, 1996), (Nee and Prasad, 1999), (Koffman and Roman, 2002). The obtained results show that the performance gain of this combined scheme relative to conventional orthogonal transmitter diversity increases with increasing the modulation level of the used linear modulation and also increases with increasing the number of branches.

The system model for orthogonal transmitter diversity is given in Section 2. In Section 3, combined Transmitter Diversity (TD) and multi-level linear modulation is introduced and discussed for Pulse Amplitude Modulation (PAM) and Quadrature Amplitude Modulation (QAM). Upper bounds on the symbol error probability of the combined schemes over both additive white Gaussian noise and Rayleigh fading chan- nels are presented in Section 4. Performance results obtained from computer simulations and supported by the bounds derived in Section 4 are presented in Section 5. Conclusions are drawn in Section 6 together with suggestions for further investigations.

\section{System Model}

Figure 1 shows the baseband representation of a conventional orthogonal transmitter diversity (frequency or time diversity) system with a total of $N$ branches. At a given symbol interval, a symbol $m$ with average energy $E_{s}$, is transmitted from the transmitter over the $N$ available branches. Assuming frequency nonselective fading multipath channel, the channel of each branch can be modeled by a complex multiplicative distortion factor. Denoting the channel coefficient of branch $i$ by $h_{i}=\alpha_{i} e^{j \theta_{i}}$, the received signal sample corresponding to the transmitted symbol $m$ can be written as follows:

$$
r_{i}=h_{i} \frac{s_{m}}{\sqrt{N}}+z_{i}, \quad i=0,1, \cdots, N-1
$$

where $\alpha_{i}$ is the fading amplitude of channel $i$ with $\overline{\alpha_{i}^{2}}=2 \sigma^{2}, \theta_{i}$ is its phase, $s_{m}$ is the baseband modulated sample of the transmitted symbol $m, z_{i}$ is complex additive white Gaussian noise random variable with zero mean and power spectral density $N_{0}$, and the factor $1 / \sqrt{N}$ comes from the fact that the total transmitted power is split between the $N$ diversity branches of the system.

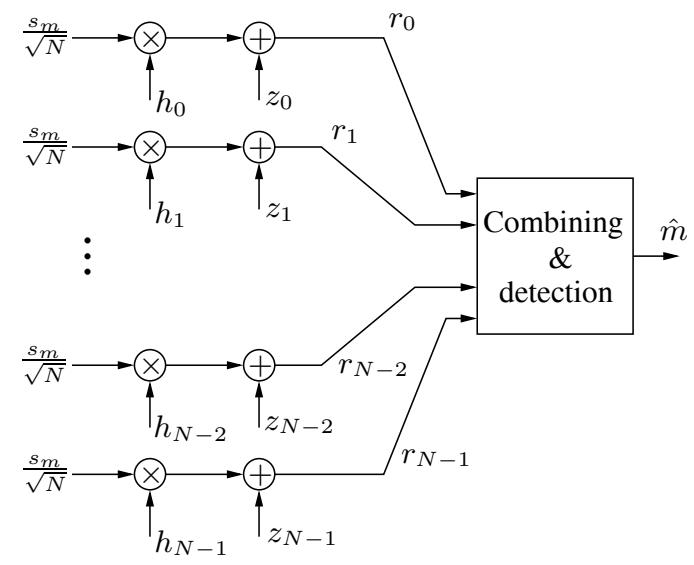

Figure 1: Principle of orthogonal transmitter diversity.

The receiver uses the received samples, computes the maximum likelihood metric of this particular symbol as follows:

$$
\mathcal{C}(m, \hat{m})=\sum_{i=0}^{N-1}\left|r_{i}-h_{i} \hat{s}_{m} / \sqrt{N}\right|^{2}
$$

and decides in favor of the symbol that gives the smallest metric. An error will occur if and only if a symbol 
$\hat{m} \neq m$ within the modulation signal set gives a metric smaller than that given by the actual transmitted symbol $m$. In this case, assuming ideal Channel state Information (CSI), the pairwise error probability ${ }^{1}$ can be written as follows:

$$
\begin{aligned}
P_{2}\left(s_{m} \rightarrow \hat{s}_{m}\right) & \leq \frac{1}{2}\left(\frac{1}{1+\frac{2 \sigma^{2}\left|s_{m}-s_{\hat{m}}\right|^{2}}{4 N_{0}}}\right)^{N} \\
& \leq \frac{1}{2}\left(\frac{1}{\frac{\left|s_{m}-s_{\hat{m}}\right|^{2}}{4 E_{b}}}\right)^{N} \frac{1}{\gamma_{0}^{N}}
\end{aligned}
$$

where $\gamma_{0}=2 \sigma^{2} E_{b} / N_{0}$ is the average Signal-to-Noise Ratio (SNR).

It is easy to verify that (2) is equivalent to a Maximum Ratio Combining (MRC) of the received signal samples followed by regular symbol-by-symbol detection (Prakis, 2001), (Alamouti, 1998). Meaning that, at every symbol interval, the received signal samples are first linearly combined to give

$$
\begin{aligned}
y & =\sum_{i=0}^{N-1} h_{i}^{*} r_{i} \\
& =\frac{1}{\sqrt{N}}\left(\sum_{i=0}^{N-1} \alpha_{i}^{2}\right) s_{m}+\sum_{i=0}^{N-1} h_{i}^{*} z_{i}
\end{aligned}
$$

which is then used to detect the symbol $m$.

Maximum ratio combining uses the combining coefficients that maximizes the signal-to-noise ratio of the combined signal. From (4), the received instantaneous Signal-to-Noise Ratio (SNR) of the combined signal can be written as follows:

$$
\Gamma=\left(\sum_{i=0}^{N-1} \alpha_{i}^{2}\right) \frac{E_{s}}{N N_{0}}
$$

which is basically the sum of the SNRs on the different diversity branches.

When the diversity branches are completely uncorrelated a diversity gain of order $N$ is achieved with this scheme (Proakis, 2001). As the number of branches increases, the sum of the fading coefficients in (5) approaches $N$ and in the limit the instantaneous SNR converges to $E_{s} / N_{0}$. Hence, it can be shown that in the limit as $N \rightarrow+\infty$, the performance of maximum ratio combining approaches that obtained over an equivalent ideal channel with AWGN only, i.e., with the same noise power $N_{0}$, but no fading. This shows that diversity is a very efficient scheme for wireless communications and can, infact, transform a fading multipath channel into an ideal channel with signal time availability of around $100 \%$.

When the communication channel is ideal with AWGN only, the multiplicative coefficients are then

\footnotetext{
${ }^{1}$ the probability of choosing the symbol $\hat{m}$ instead of $m$ given that the symbol $m$ was actually transmitted.
}

deterministic and equal with $\alpha_{i}=1, \forall i$, and the received signal-to-noise ratio after signal combining becomes

$$
\Gamma=\frac{E_{s}}{N_{0}}
$$

which is independent of the number of branches $N$. Hence, the performance of orthogonal transmitter diversity with maximum ratio combining on AWGN channels is identical to that of one branch and thus, it does not give any advantages over the nondiversity scheme and does not depend on the number of branches. This indicates that when the correlation between the different branches increases, the performance of conventional orthogonal transmitter diversity deteriorates and we may end up with nothing in return for the bandwidth expansion (frequency diversity) or efficiency loss (time diversity). When designing a diversity scheme one should make sure that the scheme will operate well over different environments and not only when the diversity branches are perfectly uncorrelated. Diversity techniques provide an extra dimension to the communication system that should be exploited well and preferably in most environments.

\section{Combined Transmitter Diver- sity and Multi-Level Modula- tion}

Let us consider the case of 4-level Pulse Amplitude Modulation (4PAM) scheme with the signal constellations as shown in Figure 2 where gray mapping has been assumed. Now, if this modulation scheme is used with a conventional orthogonal transmitter diversity with signal combining according to (2) then the signal constellations of the combined signal becomes two-dimensional as shown in Figure 3. Note that the signal space still appears as a one dimensional (identical to that of Figure 2) signal space even though we are using two dimensions. In fact, this observation is true for any number of branches where the signal space dimension of the used modulation scheme stays always the same regardless of the number of diversity branches used. This explains why orthogonal transmitter diversity does not give any advantages when there is no difference between the channel amplitudes of the different channel branches. Hence, conventional orthogonal transmitter diversity does not take full advantage of the system dimension (which is two in the case of Figure 3). Taking advantage of the available system dimension we may improve the system performance as compared to the non-diversity case under any channel conditions. The increase in the dimension of the signal space of the combined signal provides more room for the signal points of the modulation scheme. This extra room makes it possible to increase the distance between the signal points and hence improving the bit error probability of the system over AWGN channels 
and fading channels. For instance, with a simple manipulation, the signal constellations of the combined signal of Figure 3 can be optimized to give the signal constellations of Figure 4. We can easily see that the obtained signal points are now better spread in this augmented signal space with a minimum squared Euclidean distance between the signal points $4 \mathrm{~dB}$ better than that of the conventional case (Figure 3). Here, the result of Figure 4 indicates that in order to take full advantage of the augmented signal space obtained through orthogonal transmitter diversity, one has to use different signal constellations on the different diversity branches. For the case of a combined two branch transmitter diversity and 4PAM, one should use the constellations $\{00,01,11,10\}$ on the first branch and the constellations $\{11,00,10,01\}$ on the second branch. With a higher number of branches different signal constellations for the different branches can be obtained by inspection as done in Figure 4 or through computer search. With computer search, one tries to maximize the minimum squared Euclidean distance between the different signal points of the used modulation scheme through different combinations and then selects the set of constellations that gives the best result. Note that this combined scheme does apply to binary modulation since binary modulation can be optimized within one-dimensional signal space.

With this combined scheme, the receiver uses a modified version of the metric given in (2) in the signal detection. Denoting by $s_{m}^{(i)}$ the baseband modulated sample of symbol $m$ at branch $i$, the modified metric becomes:

$$
\mathcal{C}(m, \hat{m})=\sum_{i=0}^{N-1}\left|r_{i}-h_{i} \hat{s}_{m}^{(i)} / \sqrt{N}\right|^{2} .
$$

At the receiver side, the set of samples, $\left\{s_{\hat{m}}^{(i)}\right\}$, that minimizes the above metric is selected as the transmitted set.

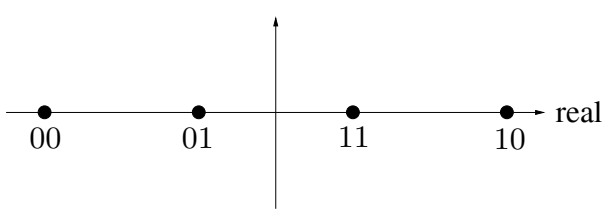

Figure 2: Signal constellations of 4PAM.

\section{Performance Analysis}

Using the metric given in (7), the detector will make an error if it chooses the symbol $\hat{m}$ given that the symbol $m \neq \hat{m}$ was transmitted. This error event will occur with probability

$$
P_{2}(m \rightarrow \hat{m})=P(\mathcal{C}(m, m)>\mathcal{C}(m, \hat{m}))
$$

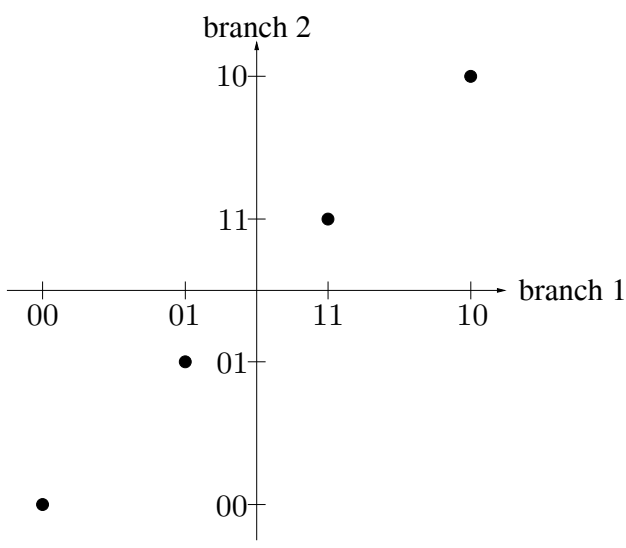

Figure 3: Signal constellations of the combined signal for conventional transmitter diversity with 4PAM and MRC combining.

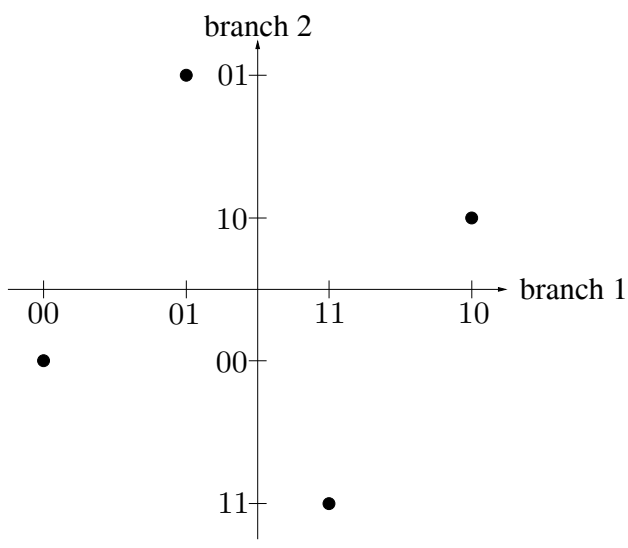

Figure 4: Signal constellations of the combined signal for combined transmitter diversity with 4 PAM and MRC combining.

For an AWGN channel, the above pairwise error probability becomes:

$$
P_{2}(m \rightarrow \hat{m})=Q\left(\sqrt{\frac{D^{2}\left(s_{m}, s_{\hat{m}}\right)}{2 N_{0}}}\right)
$$

where

$$
Q(x)=\frac{1}{\sqrt{2 \pi}} \int_{x}^{+\infty} e^{-t^{2} / 2} d t
$$

is the $Q$-function and

$$
D^{2}\left(s_{m}, s_{\hat{m}}\right)=\frac{1}{N} \sum_{i=0}^{N-1}\left|s_{m}^{(i)}-s_{\hat{m}}^{(i)}\right|^{2}
$$

is the squared Euclidean distance between the transmitted sequence $\left\{s_{m}^{(i)}\right\}$ and the sequence $\left\{s_{\hat{m}}^{(i)}\right\}$ selected by the detector.

Averaging over all possible candidate symbols and all transmitted symbols, an upper bound on the average symbol error probability of the combined scheme can 
be derived and is given by

$$
P_{s} \leq \frac{1}{M} \sum_{m=1}^{M-1} \sum_{\hat{m} \neq m} Q\left(\sqrt{\frac{D^{2}\left(s_{m}, s_{\hat{m}}\right)}{2 N_{0}}}\right)
$$

where $N$ is the number of branches. Clearly, the symbol error probability of the combined system is dependent on the signal sets used on each branch, the number of branches, and the modulation level.

For instance, for the case of 16QAM scheme, the modulation sets can be determined following the procedure described in the previous section and an upper bound on the symbol error probability can be derived and is given by

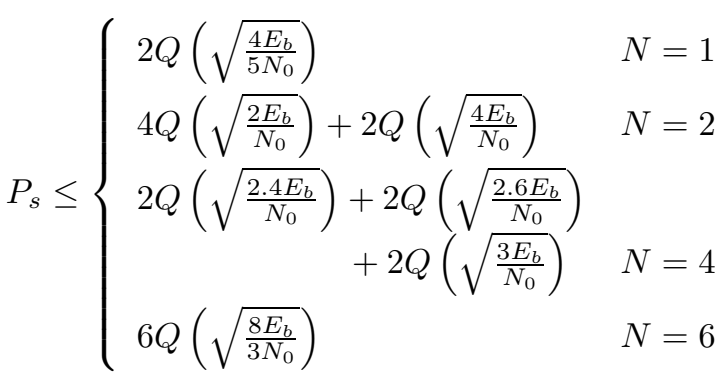

Note that when the number of branches increases the Squared Euclidean distance between the different signal points of the combined signal become more balanced and in the limit all the signal points become equidistant from each other in the new augmented signal space (similar to orthogonal signaling schemes). The above expression shows that at $N=6$, the signal points of the inphase (or quadrature) component of the 16QAM scheme are all at the same distance from each other.

For Rayleigh fading channels and with uncorrelated branches, an upper bound on the symbol error probability of combined transmitter diversity and multi-level modulation can be derived in a manner similar to the case of the AWGN channel.

For a given fading channel observation, an upper bound on the symbol error probability of combined transmitter diversity and multi-level modulation can be written as

$$
P_{s}(\mathcal{E} \mid \boldsymbol{h}) \leq \frac{1}{M} \sum_{m=1}^{M-1} \sum_{\hat{m} \neq m} Q\left(\sqrt{\frac{D_{\boldsymbol{h}}^{2}\left(s_{m}, s_{\hat{m}}\right)}{2 N_{0}}}\right)
$$

where

$$
D_{\boldsymbol{h}}^{2}\left(s_{m}, s_{\hat{m}}\right)=\frac{1}{N} \sum_{i=0}^{N-1} \alpha_{i}^{2}\left|s_{m}^{(i)}-s_{\hat{m}}^{(i)}\right|^{2}
$$

is the weighted squared Euclidean distance between the transmitted sequence $\left\{s_{m}^{(i)}\right\}$ and the sequence $\left\{s_{\hat{m}}^{(i)}\right\}$ selected by the detector and

$$
\boldsymbol{h}=\left\{h_{0}, h_{1}, \cdots, h_{N-1}\right\}
$$

is the set of fading coefficients during the observed symbol interval.

Averaging the above expression over the probability density functions of the fading coefficients, the upper bound on the symbol error probability over Rayleigh fading channels becomes (Slimane and LeNgoc, 1995)

$$
\begin{aligned}
P_{s} \leq \frac{1}{M} & {\left[\sum_{j=1}^{N}\left(\begin{array}{c}
2 N-j-1 \\
N-1
\end{array}\right) \frac{2^{j-2 N}}{\left(1+x_{\min }\right)^{2}}\right] } \\
& \times \sum_{m=1}^{M-1} \sum_{\hat{m} \neq m} \prod_{i=0}^{N-1}\left(\frac{1}{1+\frac{2 \sigma^{2}\left|s_{m}^{(i)}-s_{\dot{m}}^{(i)}\right|^{2}}{4 N N_{0}}}\right)
\end{aligned}
$$

where

$$
x_{\min }=\min _{\forall m \neq k} \sqrt{\frac{2 \sigma^{2}\left|s_{m}-s_{k}\right|^{2} /\left(4 N_{0}\right)}{N+2 \sigma^{2}\left|s_{m}-s_{k}\right|^{2} /\left(4 N_{0}\right)}}
$$

which has the same diversity order as that of the regular case (see eq. (3)) but a product distance that depends on the selected set. When the modulation signal constellations sets are properly selected, a better product distance is obtained and the overall system performance is improved.

Applying the above upper bound to the 16QAM scheme we get

$$
\begin{aligned}
P_{s} \leq & {\left[\frac{1}{1+x_{1}}\right] \frac{1}{1+0.4 \gamma_{0}}, \quad N=1, } \\
P_{s} \leq & {\left[\frac{1}{1+x_{2}}+\frac{1}{\left(1+x_{2}\right)^{2}}\right] \frac{1}{1+0.2 \gamma_{0}} } \\
& \times\left[\frac{1}{1+0.8 \gamma_{0}}+\frac{0.5}{1+1.8 \gamma_{0}}\right], N=2,
\end{aligned}
$$

where

$$
x_{N}=\sqrt{\frac{0.4 \gamma_{0}}{N+0.4 \gamma_{0}}}, \text { and } \gamma_{0}=\frac{2 \sigma^{2} E_{b}}{N_{0}}
$$

Similar calculations can be done for larger number of branches and for higher levels MQAM schemes.

\section{Simulation Results}

To illustrate the performance of combined orthogonal transmitter diversity and multi-level modulation we considered 16-level Quadrature Amplitude (16QAM) and 64QAM modulations and different number of branches. The fading multipath channel is assumed slowly varying Rayleigh distributed and uncorrelated between the diversity branches. We will also consider the system performance under ideal conditions where the transmitted signal is affected by additive white Gaussian noise only. 
Figure 5 shows both simulation results and upper bounds for the average symbol error probability of combined TD 16QAM over additive white Gaussian noise channels as a function of $E_{b} / N_{0}$ and for different number of branches. It is observed that by increasing the number of branches, better error probability performance is obtained. Compared to the conventional transmitter diversity case ${ }^{2}$, a gain of about $4 \mathrm{~dB}$ is obtained when two branches are used. This gain is increased to about $4.7 \mathrm{~dB}$ when using four branches. We also notice that the upper bound on the average symbol error probability is very tight and can be used to predict the performance of combined transmitter diversity $M Q A M$ without the need for extensive simulations.

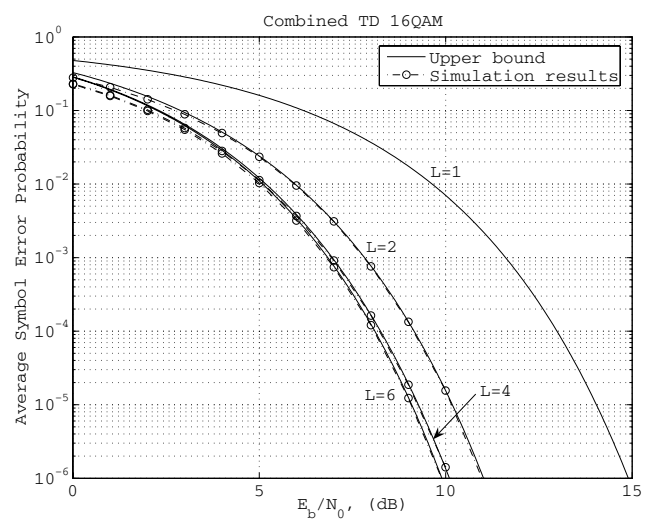

Figure 5: Average symbol error probability of combined TD 16QAM over AWGN channels and for different number of branches.

This obtained power gain can be used to improve the bandwidth efficiency of orthogonal TD systems. We know that a two branch combined TD 16QAM has the same bandwidth efficiency as uncoded QPSK modulation ( 2 bits $/ \mathrm{s} / \mathrm{Hz}$ ), and a four branch combined TD 16QAM has the same bandwidth efficiency as uncoded BPSK modulation ( $1 \mathrm{bit} / \mathrm{s} / \mathrm{Hz}$ ). Figure 6 shows that a two branch combined TD 16QAM has a similar bit error probability as QPSK modulation and a four branch combined TD 16QAM outperforms BPSK by about 1 $\mathrm{dB}$. Hence, by using this combined diversity scheme we can compensate for the efficiency loss that exists in conventional orthogonal TD schemes with almost no increase in complexity.

Figure 7 illustrates the average bit error probability of the combined scheme over Rayleigh fading channels as a function of $E_{b} / N_{0}$ and for different number of branches. Also included in the figure is the bit error probability of conventional transmitter diversity. We notice that both schemes have the same diversity order but the combined transmitter diversity scheme outperforms the conventional scheme due to the nice spread of the signal constellations of the combined scheme that gives a larger product distance. The upper bound

\footnotetext{
${ }^{2}$ As indicated earlier, the performance of conventional orthogonal TD over AWGN channels is identical to that of the non-diversity case (one branch only) regardless of the number of branches.
}

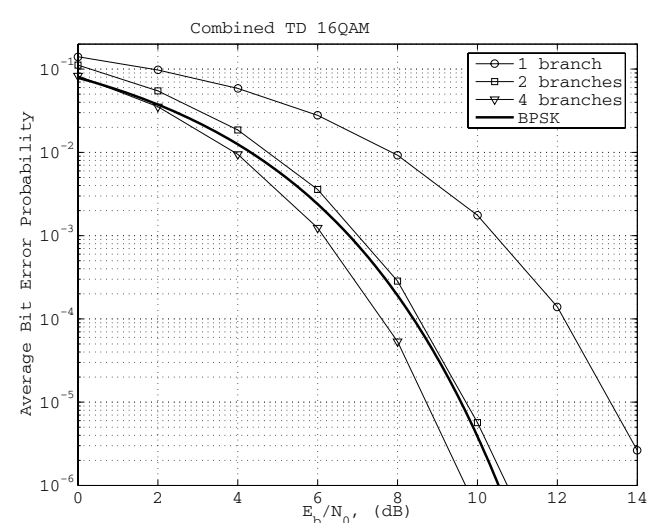

Figure 6: Performance comparison of the combined TD scheme to non-diversity schemes that have the same bandwidth efficiency over AWGN channels.

on the symbol error probability of the combined TD 16QAM is illustrated in Figure 8 for different number of branches. We notice that these upper bounds are very tight for fading channels as well.

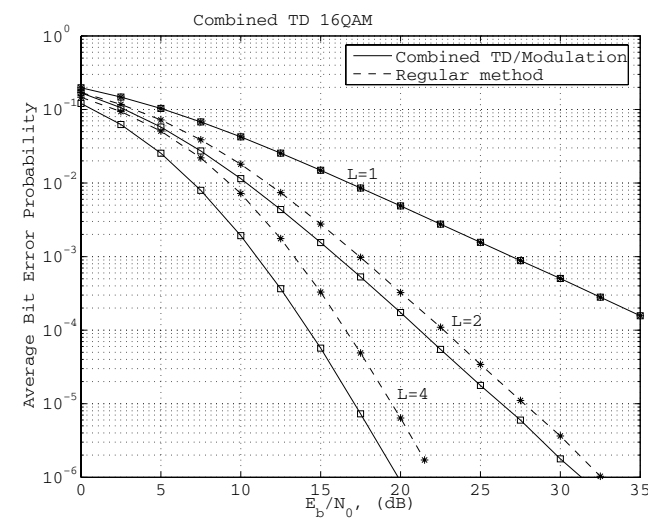

Figure 7: Average bit error probability of combined TD 16QAM over Rayleigh fading channels and for different number of branches.

Figure 9 illustrates the average bit error probability of combined TD 64QAM over AWGN channels as a function of $E_{b} / N_{0}$ and for different number of branches. We notice the same trend here where the average bit error probability improves when increasing the number of branches. However, the performance gain as compared to the conventional case is more pronounced here. For instance, two branch combined TD 64QAM performs about $7 \mathrm{~dB}$ better than conventional TD and six branch combined TD 64QAM performs about $9 \mathrm{~dB}$ better than the conventional TD scheme. We also notice that the bit error probability of six branch combined TD 64QAM is comparable to that of QPSK which shows the possibility of improving the bandwidth efficiency as compared to conventional TD schemes. The upper bound on the symbol error probability of combined transmitter diversity and 64QAM on AWGN channels is illustrated in Figure 10 where 


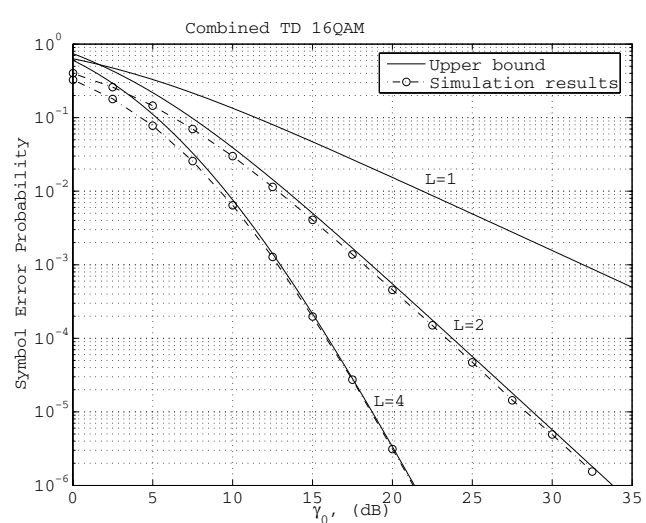

Figure 8: Average symbol error probability of combined TD 16QAM over Rayleigh fading channels and for different number of branches.

we notice a perfect match with the simulation results.

Figure 11 shows the bit error of combined TD 64QAM over Rayleigh fading channels where we notice that considerable performance gain as compared to conventional TD has been obtained.

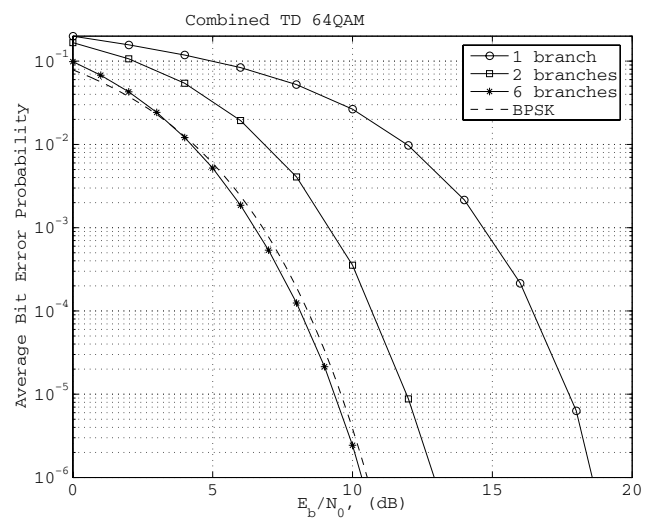

Figure 9: Average bit error probability of combined TD 64QAM over AWGN channels and for different number of branches.

Figure 11 shows the bit error of combined TD 64QAM over Rayleigh fading channels where we notice that considerable performance gain as compared to conventional TD has been obtained.

\section{Conclusions}

This paper discussed the use of multi-level linear modulation in orthogonal transmitter diversity such as frequency diversity and time diversity. Multi-level linear modulation techniques are bandwidth efficient but they are not power efficient. The lack of power efficiency of multi-level modulation is due to the limited dimension of its signal space which is less or equal to two. When used with orthogonal transmitter diversity,

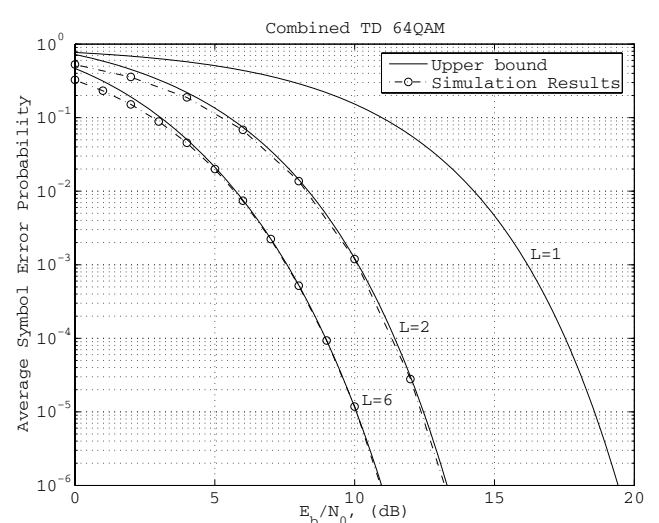

Figure 10: Average symbol error probability of combined TD 64QAM over AWGN channels and for different number of branches.

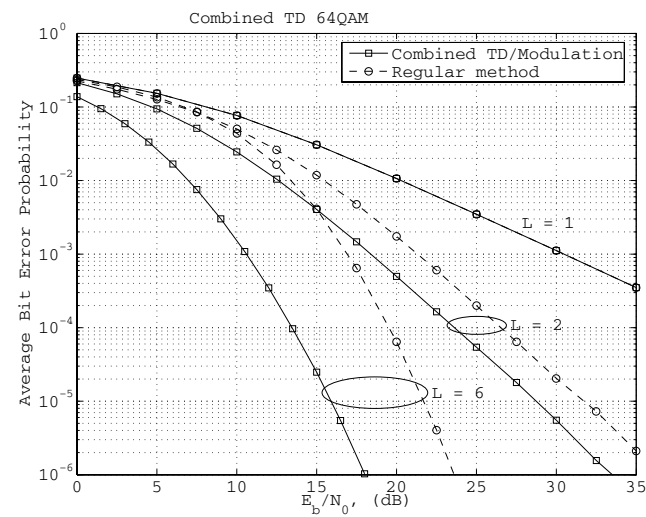

Figure 11: Average bit error probability of combined TD 64QAM over Rayleigh fading channels and for different number of branches.

there is a possibility for increasing its signal dimension and a potential for improving its power efficiency. By viewing the modulation dimension and the branches of the diversity scheme as an augmented signal space for the modulation signal points, good bit error rate performance over both AWGN channels and Rayleigh fading channels has been obtained. The obtained simulation results and upper bounds showed that this performance gain increases with the number of branches and with the modulation level. Hence, this combined scheme can be used to solve the problem of bandwidth efficiency loss seen in regular orthogonal transmitter diversity without any increase in receiver complexity. It can also provides good power saving even when the diversity branches of the wireless system are very correlated.

The results presented in this paper are for uncoded linear modulation schemes only. Combined Trellis Coded Modulation (TCM) schemes and transmitter diversity and the interaction between coding and diversity branches is a natural extension of this work. 


\section{References}

S. M. Alamouti, "A simple transmit diversity technique for wireless communications," IEEE J. Selected Areas Commun., Vol. 16, No. 8, pp. 14511458, October 1998.

A. A. Ali and I. Al-Kadi, "On the use or repetition coding with binary digital modulation on mobile channels," IEEE Trans. Veh. Technology, Vol. 38, No. 1, pp. 14-18, February 1989.

E. Biglieri, D. Divsalar, P. J. McLane, and M. K. Simon, Introduction to Trellis-Coded Modulation with Applications, Macmillan Publishing Company, 1991.

ETSI, "Broadband radio access networks (BRAN); HIPERLAN Type 2; Physical (PHY) layer", ETSI TS 101 475, v1.1.1, April 2000.
ETSI, "Digital Video Broadcasting (DVB); framing structure, channel coding and modulation for digital terrestrial television (DVB-T)," (Final Draft) prevision ETS 300 744, November 1996.

I. Koffman and V. Roman, "Broadband wireless access solutions based on OFDM access in IEEE 802.16," IEEE Commun. Magazine, pp. 96-103, April 2002.

R. V. Nee and R. Prasad, OFDM for Wireless Multimedia Communications, Arthech House Publishers, December 1999.

John G. Proakis, Digital Communications, fourth edition, McGraw-Hill, 2001.

S. B. Slimane and T. Le-Ngoc, "Tight bounds on the error probability of coded modulation schemes in Rayleigh fading channels," IEEE Trans. Veh. Technology, Vol. 44, No. 1, pp. 121-130, February 1995. 